\title{
EMANCIPACIÓN Y REPRODUCCIÓN SOCIAL EN LA EDUCACIÓN. LA EXPERIENCIA DE JACOTOT Y LA ENSEÑANZA MODERNA.
}

Leonardo Colella*

Resumen: En este artículo se busca caracterizar la educación desde las variables de "emancipación" y "reproducción social", a través de los aportes de Jacques Rancière. Para ello, se reconstruye el contexto de la enseñanza moderna en el que Jacotot despliega su propuesta pedagógica en torno a la "igualdad de las inteligencias".

Palabras clave: Emancipación, Igualdad de las inteligencias, Reproducción, Ilustración, Jacotot

Resumo: Este artigo procura delinear a educação a partir dos conceitos de "emancipação" e "reprodução social", por meio das contribuições de Jacques Rancière. Para este fim, reconstrói o contexto de ensino moderna e projeto pedagógico de Jacotot basado em a "igualdade das inteligências".

Palavras-chave: Emancipação, Igualdade das inteligências, Reprodução, Ilustração, Jacotot.

\footnotetext{
* Profesor Universitario en Filosofía y Becario doctoral del Consejo Nacional de Investigaciones Científicas y Técnicas. E-mail: leonardojcolella@yahoo.com.ar
}

\section{Introducción}

Si bien ya puede hallarse en Platón un vínculo estrecho entre la paideia y el desarrollo de la polis (JAEGER, 2001; DI CARLO, 2003), es en la modernidad donde se registra con mayor notoriedad la tensión entre una educación individual pretendidamente emancipadora y una educación destinada a la transmisión cultural para asegurar la cohesión social. Quizá sea Rousseau (WASKMAN, 2009) el caso paradigmático en el que se evidencie de manera más clara, a la vez, una noción de educación particular y doméstica, destinada a la formación integral del individuo a lo largo de su vida (plasmada en Emilio), y otra concepción de educación pública dirigida principalmente a la formación de la patria o la nación (desarrollada tiempo después en Consideraciones sobre el Gobierno de Polonia).

COLELLA, Leonardo. Emancipación y reproducción social en la educación. La experiencia de Jacotot y la enseñanza moderna. Revista Sul-Americana de Filosofia e Educação. Número 21: nov/2013-abr/2014, p. 33-46. 
Asimismo, Kant (1993) actualiza el problema entre un sujeto emancipado que hace uso de su propia razón y un sujeto gobernable que debe "obedecer", cristalizando aquella tensión a través de la distinción de los usos público y privado de la razón, y otorgándole ciertas libertades y ciertos límites según el caso.

Con todo, la educación moderna desliza hasta la actualidad una problemática que se inscribe entre sus propios fundamentos. ¿Cómo es posible que la educación suponga la formación de individuos gobernables a través de la incorporación de contenidos prefigurados $y$, a la vez, admita para sí la tarea de constitución de sujetos emancipados con capacidad para intervenir en los saberes establecidos? ¿Qué significaría que la educación, que se propone como transmisora de conocimientos, admita un lugar para pensar algo novedoso?

El nuevo escenario político y social configurado a partir de la Revolución Francesa impulsó un novedoso proyecto de educación que, en sus fundamentos principales, se sostendrá hasta la actualidad. La promoción de la industria, la República, la opinión pública y la prensa requirieron un amplio sistema de enseñanza graduado, nacional y profesional, y un método acelerado para la instrucción de las masas: la explicación. En última instancia, existía la creencia de que la emancipación requería del método explicativo para que el individuo, a través de la incorporación de diversas ciencias, pudiese servirse de ellas para poder actuar sin la tutela de otros.

Más allá de las variaciones particulares ocurridas en los últimos siglos, la educación actual continúa fundada sobre la lógica de la explicación: se trata de la estructura pedagógica que delimita un poseedor del saber (el docente), un desposeído de ese mismo saber (el alumno) y la implementación de una lógica de transmisión gradual de estos contenidos para que el segundo intente igualar los saberes del primero (HOUSSAYE, 1988; COLL, 1997; FENSTERMACHER, 1999; BERNAL, 2007).

Jacques Rancière, en Le Maître ignorant. Cinq leçons sur l'émancipation intellectuelle (1987), analiza la experiencia educativa de Joseph Jacotot, un pedagogo francés que a principios del siglo XIX desafía el mé- 
todo explicativo y con él los principios, aún en construcción, de la educación moderna.

La explicación configura un problema al interior de la educación que se pretende emancipadora al constituirse en un modo de subjetivación. En primer orden, el estudiante es definido como un "desigual" al encontrarse en un lugar de carencia (con respecto a determinados saberes y habilidades) y de imposibilidad en la toma de diversas decisiones (sobre la forma, el contenido, las condiciones y el sentido de su aprendizaje). E efecto de la educación que se proclama emancipadora podría ser paradojal al contribuir en la construcción subjetiva de aquel que carece de ciertas capacidades o de aquel que delega sus decisiones en otros, demarcando un campo jerárquico de orden intelectual según un determinado régimen de visibilidad y del uso de la palabra: aquellos que son tenidos en cuenta y aquellos que no son visualizados, aquellos que pueden articular un discurso sobre su propia realidad y aquellos que sólo emitirían señales de aceptación o rechazo, etc.
En segundo término, el problema es de orden vincular. La lógica de la explicación, al partir de una desigualdad que intenta subsanar (la disparidad entre los conocimientos y habilidades del maestro y del alumno), obtura la construcción subjetiva de una experiencia colectiva al dividir las partes vinculadas afirmando la capacidad intelectual de una (la del profesor) por sobre la incapacidad "provisoria" de la otra (la del alumno y "futuro" sujeto crítico, emancipado, etc.). De este modo, la lógica de poseedores y desposeídos instituye $y$ reproduce un escenario de segmentación jerárquica que podría contribuir a un modelo de sociedad desigualitaria: la explicación trascendería el ámbito educativo para constituirse como el vínculo mismo del orden social, esto es, como ficción distributiva y justificadora de rangos o lugares.

\section{La "formación": entre el hombre y la sociedad}

A lo largo de la historia, las reflexiones sobre la educación evaluaron diversos aspectos más preponderantes que otros en relación a ciertas temáticas consideradas por cada una 
como sustanciales y postergaron o trataron más lateralmente algunos otros; si bien difícilmente encontremos perspectivas que niegan en su totalidad algún aspecto que para otro enfoque es elemental, podemos discernir básicamente, hasta principios del siglo XX, dos grandes tendencias según la dimensión específica a la que se le otorgue mayor preponderancia: una idea de la educación que apunta a la formación individual del hombre, y otra, que más bien resalta su implicancia social (variables que paralelamente se presentan asociadas a la tensión entre la "autonomía" y el "gobierno" de los individuos)

En este sentido, CIRIGLIANO (1967) afirma que existen dos grandes tendencias identificadas en los estudios sobre educación que son heredadas por el inicio del siglo XX: la educación como "concepto" y la educación como "hecho". La primera de ellas, atribuida a los filósofos, destacaría la función de poner en contacto al individuo con la cultura. Supone poder alcanzar, a través de la transmisión cultural, un desarrollo de la personalidad $\mathrm{y}$, proyectando un ideal de hombre a conquistar, le atribuye a la educación la tarea de "perfeccionar" al individuo según aquél criterio. Aquí se inscribirían aquellos filósofos que han destacado alguna función esencial del hombre y que esperaban que la educación cumpliera con el desarrollo de esa capacidad sustancial, como el caso de Aristóteles y el ejercicio de la "virtud", los escolásticos en relación a la "trascendencia", Rousseau y su preocupación por resguardar o recuperar la "bondad natural" o la Ilustración con respecto a la potencia de la "razón". Algunos pensadores constituirían excepciones, como el caso de Platón, quien traza de forma explícita una relación entre polis y paideia (ABBAGNANO y VISALBERGHI, 1992; JAEGER, 2001).

La segunda tendencia, adjudicada a los científicos (en especial a los sociólogos), desplaza la interioridad del hombre del centro de observación y resalta el hecho de la incorporación de los nuevos individuos a la estructura social, mediante la transmisión cultural, en función de la demanda de reproducción del propio sistema. El primer exponente de la educación como "hecho" social fue Durkheim. 
Cirigliano, influido principalmente por la obra de Heidegger, propone en la década de 1960 una tercera posición que denomina "fenomenológica" (o la consideración de la educación como "fenómeno"), que intenta resignificar la relación entre "hombre" y "sociedad", y aplicarla para el caso del análisis de la educación. Admite que el fin de la educación es el "ser social", lo que significaría, en sus propios términos, que el propósito general de ésta es la democracia, y consecuentemente, el "cambio", considerado específicamente como avance o progreso social (CIRIGLIANO, 1967: pp. 92-93).

Estas transformaciones en la concepción de la educación, traen consigo una variación de orden gnoseológico y epistemológico, que será pertinente considerar en relación al concepto de "igualdad de las inteligencias" de Jacques Rancière que expondremos posteriormente. Se trata de la diferencia entre, por un lado, la consideración de la "idea" como "conocimiento", la reducción del patrimonio cultural de la sociedad a un conjunto de ideas susceptibles de ser transferidas generacionalmente, y la asociación directa entre "inteligencia" y memoria; y por el otro, la significación de la "idea" como esquema o esbozo para la acción, la inteligencia como herramienta para resolver los problemas generados en el medio social, y la "experiencia" como saldo de la acción y pieza fundamental en los aprendizajes. Los primeros rasgos descriptos abarcarían dos etapas: el alejandrinismo que se extiende desde los griegos hasta el siglo XVII, que presenta una enseñanza más bien verbalista, de fundamento intelectual y asociada a la psicología de las facultades, y el naturalismo, como momento de transición, que comprende a Comenio, Rousseau, Pestalozzi y Herbart hasta el surgimiento de las primeras escuelas nuevas, y que proponen básicamente una enseñanza de corte intuitiva, de fundamento sensorial, y apoyada en las psicologías senso-empiristas y asociacionistas. Los segundos rasgos presentados se corresponderían con planteos de diversos exponentes de la escuela nueva e incluirían a teóricos como Dewey, Whitehead y Mannhein.

La línea alemana posterior a Herbart se encuentra marcadamente 
influenciada por Dilthey, quien a finales del siglo XIX ubica a la pedagogía dentro de las Ciencias del espíritu. El objeto de la filosofía práctica será la Bildung, la configuración del hombre y el autodesarrollo del espíritu. Se subraya la idea de que la educación es obra de la cultura, la introducción del individuo en un mundo de sentidos y de valores, y no un proceso natural de desarrollo de las potencias vitales (VÁZQUEZ, 2012).

La línea francesa, en cambio, rechaza el término "pedagogía" para disociarlo de la filosofía y propone la denominación de "ciencias de la educación". Como mencionamos anteriormente, Durkheim intenta convertir las reflexiones sobre educación en una ciencia positiva de "hechos", abandonando su función normativa de prescribir las acciones de los hombres para pasar a describir la realidad de la educación como hecho social: se desplaza desde la formación del hombre al todo social; en esta transición se podrían mencionar la pedagogía científica de Binet y Claparéde, y la pedagogía experimental de Dottrens y Simon (HOUSSAYE, 2010). Las ciencias de la educación se aso- ciarán durante el siglo XX cada vez más a la sociología de la educación, para adoptar los recorridos propios de las ciencias sociales, pasando del enfoque positivista al hermenéutico y al crítico.

Con respecto a la tradición estadounidense, Dewey (1995; 1960; 1950) intenta establecer una resignificación con respecto al sujeto educativo, en tanto propone abandonar la consideración del aprendiz como "tabula rasa" en la que el maestro debe volcar sus conocimientos, y plantea tener en cuenta el bagaje de experiencias del estudiante. En este sentido, utiliza diversas metáforas críticas, tales como "educación de cañería" o "educación fonográfica", que buscan representar una educación en la que el alumno es material de depósito o de grabación de información que luego podrá reproducir mecánicamente (PAZ DE VARGIU, 2009).

En Dewey el sujeto educativo debe ser activo ya que el aprendizaje tiene que ser impulsado por problemas de la propia experiencia. Consecuentemente, el papel del maestro deberá ser el de proporcionar estas condiciones que estimulen el contexto 
de aprendizaje y el de adoptar una postura de aprobación ante los progresos graduales de sus alumnos, integrando la psicología en los programas de estudio.

Luego de Dewey, a mediados del siglo XX, en las instituciones académicas norteamericanas, predominaban tres enfoques filosóficos sobre la educación con sus respectivas perspectivas sobre los sujetos implicados: el reconstruccionismo, que considera al educando como un ser prospectivo que se educa para la construcción del futuro, designa al currículum como el instrumental para el mundo por crear y define como método educativo la resolución de problemas; el existencialismo, para el que educar es procurar conciencia de sí mismo, en el que la figura del educador es un "provocador" y el educando es creador de su propia esencia, responsable de su libertad; y el movimiento analítico de corte positivista, para el que la educación consiste en promover una técnica para pensar y emitir juicios pertinentes, en el que la función del educador es arrojar luz sobre el proceso de comunicación y aprendizaje, y por medio del cual el estudiante debe adquirir un "método científico" a través de la incorporación de los procedimientos de descubrimiento y de validación del conocimiento, las técnicas de observación, medición, análisis estadístico, etc.

\section{La emancipación del individuo y el gobierno de la Nación}

Con respecto al planteo expuesto por Cirigliano, que traza una divergencia histórica (aunque con expresas excepciones) entre una educación dedicada al individuo y otra más concentrada en la sociedad, quizá el autor no tenga en cuenta algunos matices en principio paradójicos, especialmente, de la educación moderna. En la obra del propio Rousseau, puede rastrearse ya una variación similar entre una educación individual cuya formación abarca todos los aspectos de una vida a lo largo del tiempo (DOMINIQUE, 1988; ABAL DE HEVIE, 1982), y una educación pública que se ocupa de los destinos colectivos de una nación.

Emilio (1999) relata las experiencias entre un alumno imaginario y su tutor quien, presente desde su nacimiento, lo acompañará a lo largo de 
toda su vida con el objetivo de formarlo de manera integral por medio de una educación natural. La propuesta educativa de Rousseau se origina a partir de un presupuesto antropológico que le atribuye al hombre una bondad natural. La educación se orientaría a resguardar esta bondad que es insistentemente tentada de ser corrompida por la sociedad civil. Para ello, la tarea del maestro más que prohibir ciertas inclinaciones y prácticas debe reconstruir un vínculo próximo entre quien aprende y la naturaleza, posibilitando que éste aprenda por sí mismo, mediante los propios errores y la experimentación.

Sin embargo, diez años más tarde, en Consideraciones sobre el Gobierno de Polonia (1988), Rousseau dirigiría sus reflexiones educativas hacia la necesidad de conducir a los hombres, mediante la educación, hacia un orden político justo que garantice la libertad: "Es la educación la que debe dar a las almas la fuerza nacional, así como dirigir de tal manera sus opiniones y sus gustos que lleguen a ser patriotas por inclinación, por pasión, por necesidad. Al abrir los ojos, un niño debe ver la patria, y hasta la muerte no debe ver otra cosa" (ROUSSEAU, 1988: p. 68). En un caso o en otro, los procesos de subjetivación que se hallan concentrados en el aprendiz reflejarían aquella tensión inicial para la reflexión filosófica sobre la educación: la existente entre la formación subjetiva del "hombre" o del "ciudadano".

No obstante, si bien la filosofía moderna centra su atención en el sujeto, no descuida el problema de la "universalidad". Se trata de una reflexión que identifica al sujeto como portador de una potencia de universalidad, capaz de adoptar perspectivas generales. Existen estudios que tienen en cuenta la generalidad de la obra roussoniana y que consideran erróneas las interpretaciones sobre el autor que oscilan entre una perspectiva individualista y otra totalitaria. Por el contrario, resaltan como idea central de las propuestas rousseaunianas la caracterización del sujeto como portador de una universalidad dotada por naturaleza y con capacidad para desplegar un proceso de descubrimiento de esta presencia universal que hay en él, y que para el caso de la educación (incluso la centrada en el 
individuo), aquella capacidad subjetiva universal traería consecuencias de orden social y político que lo trascenderían como individualidad (DI CARLO Y GAMBA, 2003: pp. 92-93).

En el mismo sentido, a finales del siglo XVIII, Kant (1993) afronta la tensión aludida. Define la Ilustración como la salida del hombre de su estado de tutela. Éste sería entendido como un estado de la voluntad que demanda la aceptación pasiva de la autoridad ajena, relegando el uso de la propia razón (FOUCAULT, 1993). El conflicto entre la autonomía del sujeto y la reproducción del orden social obliga a Kant a disertar sobre la libertad y a distinguir diferentes ámbitos para razonar, y lo hace concluir en limitar el uso privado de la razón, aquel que utiliza el individuo en carácter de "funcionario", en contraposición al que hace en calidad de crítico ante el gran público, afirmando que el uso "público" de la razón debería gozar de una ilimitada libertad. De este modo, para el escenario de la educación, aquellos individuos implicados en cualquier aspecto educativo en carácter de actores institucionales y que se encuentren desempeñando una función institucional (ya sea en calidad de estudiante, docente, directivo, etc.) deberán limitar la utilización de su propia razón y "obedecer". Así, el concepto de emancipación en la Ilustración no sólo implicaría la utilización de la propia razón despojada de tutelas ajenas, sino que aquel uso sería limitado (o censurado) en el ámbito "privado".

\section{La explicación en jaque \\ Le maitre ignorant (1987) se} construye a partir de las experiencias pedagógicas de la figura de Joseph Jacotot, que a comienzos del siglo XIX y por circunstancias azarosas provocaron en él un cambio de perspectiva con respecto a la enseñanza tradicional. Jacotot se vio forzado a abandonar Francia y a dictar clases de literatura francesa en una universidad de los Países Bajos. Él no hablaba holandés y sus estudiantes no comprendían el francés. A través de una edición bilingüe de Telémaco de Fenelón, sus alumnos no sólo terminaron por comprender el texto completo, sino que realizaron ensayos críticos que contenían valiosas observaciones personales sobre la obra, 
expresados en el nuevo idioma. A ésta, se sumaron muchas otras experiencias en las que Jacotot lograba exitosamente que sus alumnos aprendieran temáticas que él no sabía. El replanteo pedagógico elaborado por Rancière-Jacotot consideraba la posibilidad de aprender sin las explicaciones de un maestro (lo que no significaba prescindir de la figura del maestro, sino renunciar a su función de "transmisor" o "explicador").

La lógica de la explicación supone un proceso de transmisión de ciertos saberes que el maestro posee y que el alumno no $y$, consecuentemente, demanda cierta subordinación intelectual que configura un escenario de segmentación jerárquica entre poseedor y desposeído. Rancière señala que las instituciones educativas modernas han adoptado el rol de reproducir esta desigualdad jerárquica hasta el punto de convertirla en su propia condición de posibilidad: de tal forma que el sentido de la escuela acabe por convertirse en la perpetua designación de desiguales-inferiores, cuyo criterio diferenciador sería la carencia de ciertos saberes y su característica distintiva la incapacidad de obtenerlos por medio propio. La función esencial del maestro explicador sería la inscripción de quienes aprenden en un circuito dominado por estructuras estamentales y por una lógica de superiores e inferiores.

A través de la experiencia de Jacotot, Rancière sugiere que es posible pensar en la construcción de una nueva relación entre maestros $y$ alumnos que no esté mediada por el recurso de la explicación. El maestro emancipador no es quien transmite su propio saber, sino quien, a través del vínculo entre voluntades, enseña al otro a ejercer su propia inteligencia. El conocimiento pierde la única dirección, antes desplegada desde el maestro hacia el alumno, para configurar nuevos y múltiples sentidos. Los saberes no constituyen un universo diferenciador (entre aquél que los posee y aquellos que no), sino que, por el contrario, asumen la forma de interrogantes compartidos que sostienen el vínculo entre el maestro y el alumno, en el que se verifica la igualdad de las inteligencias.

La propuesta de Rancière, lejos de apuntar a la comprobación de una desigualdad e intentar progresiva- 
mente a futuro disuadirla mediante la transferencia de contenidos, postula la igualdad como punto de partida para extraer de ella toda la potencia de sus consecuencias. En efecto, no se trata de corroborar la carencia de ciertos conocimientos, sino de instar al descrédito de la inferioridad de las inteligencias. Desde una perspectiva rancieriana, la inteligencia no es interpretada como la posesión de ciertos saberes o como el ejercicio de ciertas facultades intelectivas complejas, sino más bien, como la capacidad de pensar y decidir sobre la propia vida a través de la posibilidad de indagarse a uno mismo y poner en cuestión la relación que se tiene con los saberes. Esta variante de carácter gnoseológico permite afirmar que la igualdad de las inteligencias, en vez de ser un objetivo a conquistar mediante la explicación, debe ser un postulado filosófico-político inicial para toda propuesta de enseñanza que se pretenda emancipadora.

\section{La emancipación según Jacotot}

Para reconstruir el concepto de "emancipación", Rancière utiliza una definición de tipo antropológica ex- puesta por Jacotot: el hombre es una voluntad servida por una inteligencia. Un vínculo educativo entre dos individuos implica, en realidad, dos relaciones y no una sola: se trata de un vínculo del orden de la voluntad y otro del ámbito de la inteligencia. En este sentido, se denominará "emancipación" a la distancia entre estas dos relaciones, en la que una inteligencia obedece únicamente a sí misma, aunque una voluntad sea influenciada por otra.

Como hemos señalado antes, la educación puede sostenerse en el principio de la desigualdad (la explicación) o en el de la igualdad de las inteligencias. Por lo tanto, para Rancière, lo que emancipa o embrutece no es el procedimiento, los contenidos, la didáctica o el método, sino el principio que se halla detrás de todo elemento educativo.

El maestro emancipador, debe él mismo estar emancipado, esto sería reconocerse a sí mismo como partícipe de una misma potencia intelectual, común a la de todos. A esta toma de conciencia acerca de la igualdad de las inteligencias, Jacotot la denomina emancipación. Se trata de que cada 
hombre comprenda su naturaleza de sujeto intelectual. Aquí Jacotot invierte el cogito cartesiano: soy hombre, luego pienso. El pensamiento no es un atributo de la sustancia pensante, sino de la humanidad, de allí la igualdad intelectual entre los hombres. Y de allí también la nueva filosofía denominada "panecástica" que se interesa por el "todo" de la inteligencia humana en cada manifestación intelectual particular.

De este modo, el maestro no se constituye en un medio que posibilita el acceso al saber, sino que asume la tarea de mostrar, verificar y actualizar, en cada acto educativo, de lo que es capaz una inteligencia cuando se reconoce igual a cualquier otra, $\mathrm{y}$ cuando admite a cualquier otra como igual a la suya: por ello sólo un emancipado puede ser un emancipador. No es posible, en este sentido, limitar el acto emancipatorio a ningún ámbito específico, no es posible regularlo ni reglarlo, tampoco anticipar las consecuencias institucionales que de él se desprenderían, por el contrario, se trata de que siempre, a cada momento y en todo acto, se verifique el principio de la igualdad de las inteligencias.

\section{Conclusión}

Como hemos señalado, la educación ha oscilado entre la aparente pretensión de constituir individuos autónomos y la de formarlos teniendo en cuenta la reproducción del orden social. Asimismo, la educación asume la tensión provocada por la intervención creadora del sujeto que aprende y la incorporación de los saberes instituidos $\mathrm{y}$ valorados socialmente. En última instancia, asume para sí la paradójica tarea de actualizar la libertad del sujeto que a través de sus propias experiencias y la autorreflexión se constituye a sí mismo, y la libertad de la estructura social que a través de la figura del maestro busca la ligazón del lazo social, a través de la transmisión cultural.

En este sentido, la educación podría sustentarse en el principio de la libertad del maestro para "formar" a los nuevos individuos según una "forma" predefinida por las necesidades de la estructura social, o bien podría sostenerse en el principio de la libertad del aprendiz, resguardándolo 
de toda "contaminación" externa y protegiendo sus propias experiencias y capacidades individuales. Ahora bien, si se admite que la subjetividad es, en cierta medida, a la vez constituida y constituyente con respecto al orden social y cultural, ambas libertades educativas se tornarían difícilmente distinguibles. El análisis aparentemente paradójico, entre maestro $y$ alumno, no sería demasiado ajeno al problema entre "sujeto" y "estructura" que aborda la filosofía desde la década de 1970, transportado al ámbito de la educación.

El aporte brindado por Rancière para el caso de la educación, logrado a través de sus investigaciones sobre las experiencias pedagógicas de Jacotot, consistiría en la posibilidad de apartarse de la idea de libertad como la libre potencia intelectual de un cuerpo individual en un ámbito restringido; en contraposición a ello, nos invita a considerar la libertad como el reconocimiento, de todos hacia todos, de la libre participación de cada uno en una potencia intelectual (creativa) común. Esto pone de manifiesto la tensión ocasionada desde la modernidad, en el ámbito educativo, entre una concepción emancipatoria que apunta a servirse de la propia razón sin tutelas ajenas, y otra que se dirige a poner en cuestión una y otra vez la propia "razón" (y la presencia ajena que hay en ella) a través del encuentro con otros. En el mismo sentido, el sujeto de la educación no sería ni el maestro ni el estudiante, sino el vínculo construido pory entre ellos.

\section{Referencias bibliográficas}

ABAL DE HEVIA, Isabel. Historia de la Educación. Buenos Aires: Ediciones Braga, 1982.

ABBAGNANO, Nicola y VISALBERGHI, Aldo. Historia de la pedagogía. México: Fondo de cultura económica, 1992.

BERNAL, Carlos. "Un análisis crítico del modelo del triángulo pedagógico. Una propuesta alternativa". Revista mexicana de investigación educativa, Año $12, \mathrm{~N}^{\circ}$ 32, 2007, pp. 435-456.

CIRIGLIANO, Gustavo. Filosofía de la educación. Buenos Aires: Humanitas, 1967. COLL, César. ¿Qué es el constructivismo? Buenos Aires: Magisterio del Río de La Plata, 1997. 
DEWEY, John. Democracia y educación. Madrid: Morata, 1995.

DEWEY, John. La educación de hoy. Buenos Aires: Estrada, 1960.

DEWEY, John. Las escuelas de mañana. Buenos Aires: Losada, 1950.

DI CARLO, Enrique y Gamba, Ricardo. Estudios en educación. Un examen desde Platón a Piaget. Madrid: Miño y Dávila, 2003.

DOMINIQUE, Julia. "Los cambios del sistema educativo en Francia durante el siglo XVIII". En: Educación e Ilustración: dos siglos de reformas en la enseñanza: ponencias, Madrid: Centro de Investigación y Documentación educativa, 1988.

FENSTERMACHER, Gary. Enfoques de la enseñanza. Buenos Aires: Amorrortu, 1999.

FOUCAULT, Michel. "Qu'est-ce que les Lumières?”, Magazine Littéraire, $\mathrm{N}^{\circ} 309$, 1993.

HOUSSAYE, Jean. "De la naissance des philosophes de l'éducation en France". En: VERGNIOUX, Alain. (Ed.). Actes du congrès de l'Actualité de la recherche en éducation et en formation (AREF), Université de Genève, 2010.

HOUSSAYE, Jean. Le triangle pédagogique. Berna: Peter Lang, 1998.

JAEGER, Werner. Paideia: los ideales de la cultura griega. México: Fondo de cultura económica, 2001.

KANT, Immanuel. ¿Qué es la Ilustración? Madrid: Tecnos, 1993.

PAZ DE VARGIU, Sara. "Enseñar filosofía, los aportes de Dewey, Gramsci y Rorty". En: CERLETTI, Alejandro (comp.). La enseñanza de la filosofía en perspectiva. Buenos Aires: Eudeba, 2009.

RANCIÈRE, Jacques. Le maître ignorant: Cinq leçons sur l'émancipation intellectuelle. París: Fayard, 1987.

ROUSSEAU, Jean. Emilio, o de la educación. México: Porrúa, 1999.

ROUSSEAU, Jean. Consideraciones sobre el Gobierno de Polonia. Madrid: Tecnos, 1988.

VÁZQUEZ, Stella. Filosofía de la educación: estado de la cuestión y líneas esenciales. Buenos Aires: CIAFIC, 2012.

WASKMAN, Vera. "Variaciones en torno al maestro y el discípulo". En: CERLETTI, Alejandro (comp.). La enseñanza de la filosofía en perspectiva. Buenos Aires: Eudeba, 2009. 